\title{
Electrodes of supercapacitors from nanoporous carbon with nanocarbon additives
}

\author{
E. A. Kiseleva, M. A. Zhurilova, E. I. Shkolnikov \\ Joint Institute for High Temperatures, Russian Academy of Sciences, \\ Izhorskaya st. 13 Bd.2, Moscow, 125412, Russia \\ kanna787@mail.ru
}

PACS 61.46.+w

DOI 10.17586/2220-8054-2018-9-1-123-124

Electrochemical double-layer capacitors (EDLC) are emerging energy storage technology, highly demanded for rapid transition processes in transport and stationary applications, concerned with rapid power fluctuations. Rough structure of activated carbon, widely used as electrode material because of its high specific area, leads to poor electrode conductivity. Therefore there is the need for conductive additive to decrease internal resistance and to achieve high specific power and high specific energy. Usually, carbon black is widely used as conductive additives. In this paper, electrodes with different conductive additives - two types of carbon black and single-walled carbon nanotubes - were prepared and characterized in organic electrolyte-based EDLC cells. Electrodes are based on original wood-derived activated carbon produced by potassium hydroxide high-temperature activation at Joint Institute for High Temperatures RAS. Electrodes were prepared from slurry by cold-rolling. For electrode characterization cyclic voltammetry, equivalent series resistance measurements and galvanostatic charge - discharge were used.

Keywords: carbon nanotube, activated carbon, electrochemical double-layer capacitors, electronic properties.

Received: 19 June 2017

\section{Introduction}

Carbon nanotubes are a very attractive material for power source applications due to their high conductivity, chemical stability and excellent ability to form percolation clusters in an electrode structure [1]. Activated carbon is a more traditional and efficient electrode material, which allows specific capacitance of about $100 \mathrm{~F} / \mathrm{g}$ for organic electrolyte EDLC and 150-200 F/g for aqueous EDLC [2]. So high surface area can be considered as the main advantage of activated carbon as an electrode material, but high surface area usually means rough surface, and, because of large structure defects amounts, low conductivity. This leads to loss of energy, stored in EDLC and low specific power, which is the most important EDLC parameter. To increase specific power, carbon nanotubes or other conductive, but chemically-stable, electrolyte solution-soluble additive must be used in the electrode structure.

\section{Experimental}

The strategy of our approach was to use high-quality carbon nanotubes, received from OCSiAl company [3] to improve the conductivity of electrode and decrease weight percentage of carbon additive (due to high aspect ratio of these nanotubes) which doesn't participate in electric double layer formation. Some traditional additives (carbon black Vulcan XC72R and P267E (RAS Institute for Hydrocarbon Processing Problems)) were used as reference materials to derive the effect of carbon nanotubes and compare them with traditional additives. To characterize electrochemical behavior of different conductive additives, composite electrodes were prepared and tested in a supercapacitor single cell. Slurry from activated carbon, conductive additive and PTFE emulsion was prepared, containing about $8 \mathrm{wt} \%$ (dry) PTFE, $10 \mathrm{wt} \%$ (dry) carbon additive (in the case of carbon black) and $82 \mathrm{wt} \%$ (dry) of activated carbon. In the case of carbon nanotubes (TuballTM deionized water dispersion of single-walled carbon nanotubes $(0.1 \mathrm{wt} \%$ of CNT), received from OCSiAl company), dry weight of conductive additive was $0.005-0.012 \mathrm{wt} \%$ (because of addition 5-10 wt $\%$ of dispersion to slurry), and activated carbon share increased up to about 95-96 wt\%. The slurry was mechanically mixed using Ultra Turrax T10 during $10 \mathrm{~min}$, after that, solvent was partly evaporated to receive active mass which was fed to rolling press MSK-HRP-MR100DC (MTI Corp., USA) and rolled several times to obtain a composite PTFE-bound carbon sheet with 100-140 $\mu$ m thickness. A mre thorough procedure for electrode preparation is given in [4]. These sheets were annealed at $250{ }^{\circ} \mathrm{C}$ in BinderTM vacuum chamber for $4 \mathrm{~h}$ to finally remove residual water and solvent from electrode. After annealing, electrode specimens of $30 \times 30 \mathrm{~mm}$ area were prepared from sheets in Ar-filled glove box for testing in supercapacitor cells. Aluminum carbon-covered foils from MTI-Corp (USA) were used as current collectors. Cells were assembled in dry atmosphere (dry glove box filled with Ar gas) and filled with $1 \mathrm{M}$ tetraethylammonium tetrafluoroborate (TEA-BF4) in electrochemical grade (water content $<50 \mathrm{ppm}$ ) dissolved in acetonitrile (AN) purchased from BASF, USA, using cellulose-based separator. Cells were laminated to prevent moisture and impurities penetration 
from the environment was also conducted in a dry box under Ar atmosphere. To analyze carbon nanotubes content influence, three additional groups of electrodes were prepared - with $0.005,0.008$ and $0.012 \mathrm{wt} \%$ of carbon nanotubes additive.

In all experiments, activated carbon produced in Joint Institute for High Temperatures from pyrolyzed birch by alkaline activation technique based on described in [5] was used as active material of electrode.

Cyclic voltammetry (CVA) was carried out using the electrochemical analyzer VoltaLab PGZ 301, galvanostatic cycling (GC) with the current density of $10 \mathrm{~mA} / \mathrm{cm}^{2}$ and electrical impedance spectroscopy (EIS). All measurements were carried out in range from 1.25 up to $2.5 \mathrm{~V}$ of cell voltage.

\section{Results and discussion}

Results for primary GC and resistance $(R)$ measurements by voltage drop for different electrodes are given in Table 1 (after 1000 cycles).

TABLE 1. Results of primary GC for different electrode composition

\begin{tabular}{|c|c|c|c|c|}
\hline Electrode composition (conductive additive) & $C_{s p v}, \mathrm{~F} / \mathrm{cm}^{3}$ & $C_{s p m}, \mathrm{~F} / \mathrm{g}$ & $R$, Ohm & Energy efficiency, \% \\
\hline Tuball CNT, $0.005 \mathrm{wt} \%$ & 20.42 & 123 & 0.18 & 95.9 \\
\hline Tuball CNT, 0.008 wt $\%$ & 20.74 & 126 & 0.15 & 96.7 \\
\hline Tuball CNT, 0.012 wt\% & 23.8 & 152 & 0.13 & 97 \\
\hline P267E carbon black, 10 wt\% & 18.8 & 122 & 0.18 & 96.1 \\
\hline Vulcan XC72R carbon black, 10 wt\% & 19.1 & 114 & 0.23 & 95.4 \\
\hline
\end{tabular}

Table 1 data shows, that $0.005 \mathrm{wt} \%$ of single-walled carbon nanotubes is enough to reach capacity, efficiency and $R$, close to those for $10 \mathrm{wt} \%$ of carbon black. Slight increase in capacity for Tuball CNT-containing electrode comparing to carbon blacks can be explained by increase of activated carbon weight share in electrode. This additional carbon, substituting carbon black, can introduce additional capacity. Further capacity growth (with CNT mass increase) may be caused by introduction of CNT between activated carbon particles thus preventing their agglomeration. Additional pore volume is available for electric double layer formation and electrode capacity increases. Two types of carbon black, involved in experiments, demonstrate close to each other parameters with slightly higher ESR for Vulcan XC72R and slightly lower capacity for P267E, which can be explained by more rough surface structure in case of Vulcan XC72R.

\section{Conclusion}

EDLC electrodes with single-walled carbon nanotubes and two types of carbon black as conductive additives were prepared and tested in model EDLC cells with $1 \mathrm{M}$ tetraethylammonium tetrafluoroborate solution in acetonitrile as electrolyte. $0.005 \mathrm{wt} \%$ of CNT allowed us to obtain cell equivalent series resistance and energy efficiency close to those for $10 \mathrm{wt} \%$ of carbon black. Substitution of carbon black with activated carbon allowed an increase in the specific capacity of electrodes. Increase of CNT content up to $0.012 \mathrm{wt} \%$ led to a $25 \%$ increase in the electrode specific volumetric capacitance and further equivalent series resistance, possibly due to percolation cluster formation from CNT and decrease of activated carbon agglomeration degree. Thus, high-quality single-walled carbon nanotubes can be used as conductive additive to EDLC electrode instead of carbon black, increasing capacitance and decreasing resistance.

\section{Acknowledgements}

This research has been supported by Leading Scientific School NSh.8406.2016.8.

\section{References}

[1] Krause B., Potschke P., Ilin E., Predtechenskiy M. Melt mixed SWCNT-polypropylene composites with very low electrical percolation. Polymer, 2016, 98, P. 45-50.

[2] Pandolfo A.G., Hollenkamp A.F. Carbon properties and their role in supercapacitors. J. Power Sources, 2006, 157, P. 11-27.

[3] New production technology for single wall carbon nanotubes. Reinforced Plastics, 2014, 58(4), P. 19. URL: http://www.sciencedirect.com/science/article/pii/s0034361714701730.

[4] Kiseleva E.A., Lelin F.V., Zhurilova M.A., Shkolnikov E.I. Methods of forming electrodes of supercapacitors with aqueous and organs. Russ. J. Appl. Chem., 2017, 90(5), P. 712-715.

[5] Volperts A., Dobele G., et al. Supercapacitor electrodes from activated wood charcoal. Bulgar. Chem. Commun., 2016, 48, P. 337-341. 